\title{
Models of infiltration into homogeneous and fractal porous media with localized sources
}

\author{
Fabio D. A. Aarão Reis* \\ Instituto de Física, Universidade Federal Fluminense, \\ Avenida Litorânea s/n, 24210-340 Niterói RJ, Brazil \\ Vaughan R. Voller ${ }^{\dagger}$ \\ Department of Civil, Environmental, and Geo- Engineering, \\ Saint Anthony Falls Laboratory, University of Minnesota, \\ 500 Pillsbury Drive SE, Minneapolis, MN 55455, USA
}

(Dated: April 11, 2019)

\begin{abstract}
We study a random walk infiltration (RWI) model, in homogeneous and in fractal media, with small localized sources at their boundaries. In this model, particles released at a source, maintained at a constant density value, execute unbiased random walks over a lattice; a model that represents solute infiltration by diffusion into a medium in contact with a reservoir of fixed concentration. A scaling approach shows that the infiltrated length, area, or volume evolves in time as the number of distinct sites visited by a single random walker in the same medium. This is consistent with numerical simulations of the lattice model and exact and numerical solutions of the corresponding diffusion equation. In a Sierpinski carpet, the infiltrated area is expected to evolve as $t^{D_{F} / D_{W}}$ (Alexander-Orbach relation), where $D_{F}$ is the fractal dimension of the medium and $D_{W}$ is the random walk dimension; the numerical integration of the diffusion equation supports this result with very good accuracy and improves results of lattice random walk simulations. In a Menger sponge in which $D_{F}>D_{W}$ (i.e., a fractal with a dimension close to 3 ), a linear time increase of the infiltrated volume is theoretically predicted and confirmed numerically. Thus, no evidence of fractality can be observed in measurements of infiltrated volumes or masses in media where random walks are not recurrent, although the tracer diffusion is anomalous. We compare our findings with results for a fluid infiltration model in which the pressure head is constant at the source and the front displacement is driven by the local gradient of that head. Exact solutions in two and three dimensions and numerical results in a carpet show that this type of fluid infiltration is in the same universality class of RWI, with an equivalence between the head and the particle concentration. These results set a relation between different infiltration processes with localized sources and the recurrence properties of random walks in the same media.
\end{abstract}

\section{INTRODUCTION}

An infiltration process takes place when the external surface of an initially dry porous medium is in contact with water source at a fixed pressure head. In this circumstance, assuming that the Darcy law holds, the gradient of the pressure head will move moisture into and through the pore spaces, as schematically shown in Fig. 1(a). If the domain geometry constrains the moisture to a one-dimensional horizontal flow, we can invoke a Green-Ampt [1] approximation and assume a well defined moving front $S(t)$ between filled and empty pore spaces. Under this scenario, it would be expected that the filled volume $F(t)$ will increase in time as

$$
F \sim t^{n}
$$

with the infiltration exponent $n=1 / 2$ 24]. However, in many systems a different exponent is measured [5-13, which characterizes some type of anomalous transport (subdiffusive if $n<1 / 2$, superdiffusive if $n>1 / 2$ ).

\footnotetext{
* reis@if.uff.br

$\dagger$ volle001@umn.edu
}

A related process occurs when the porous medium is filled with a static fluid and the external surface is in contact with a reservoir of the same fluid but with a different concentration of a solute. This solute then infiltrates into or out of that medium, depending on the concentration difference, with the solute molecules moving diffusively; see Fig. 1(b). A model for this process in a two-dimensional lattice was proposed by Sapoval, Rosso, and Gouyet 14, and it was recently extended to fractal lattices [15]: the solute is represented by particles that execute random walks in the lattice and that have a constant concentration at an external boundary. Here, this is termed random walk infiltration (RWI). Ref. 15. used a scaling approach and numerical simulations to show the relation between the exponent $n$, the fractal dimensions of the infiltrated medium $\left(D_{F}\right)$ and of the infiltration boundary $\left(D_{B}\right)$, and the random walk dimension in that medium $\left(D_{W}\right)$.

In this work, we study RWI in homogeneous and fractal media with a small source at an external boundary and discuss the close relation between this problem and fluid infiltration in the same media. For two- and threedimensional media, the time evolutions of the infiltrated area and volume are obtained from the solution of the diffusion equation and confirmed by numerical simulations. 
(a)

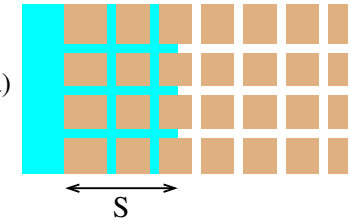

(b)

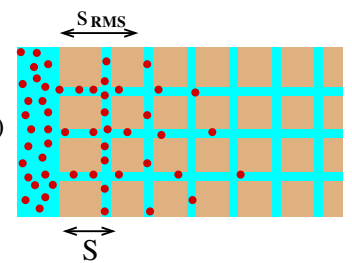

FIG. 1. (a) Schematic of fluid (blue) infiltration from a high pressure reservoir at the left into a porous media (solid in tan color, non-filled pores in white). (b) Infiltration of a solute (red) from a reservoir at the left into a porous media initially without that solute. The characteristic lengths $s_{R M S}$ and $S$ are indicated for one-dimensional infiltration.

A scaling approach is then used to show that the infiltration has the same time evolution of the number of distinct sites visited by a single random walker in the infiltrated lattice. This leads to estimates of the infiltration exponent in fractal media, including the prediction of an apparently normal infiltration in fractals where $D_{F}>D_{W}$, as illustrated for a Menger sponge. In fluid infiltration, where the sharp fluid front moves with a velocity proportional to the pressure gradient, the analytical form of the time evolution of the infiltrated area or volume in two- and three-dimensional media exactly matches that seen in geometrically equivalent RWI processes. Additional numerical simulations also reveal similarities for fluid infiltration and RWI in Sierpinski obstacle carpets. Together these results confirm the scaling equivalence of fluid infiltration with RWI, and establish a connection between different infiltration models with localized sources and the recurrence properties of random walks.

Despite the simple features of the fractal media studied here, we recall that they were used in recent laboratory studies of anomalous transport. For instance, infiltration of a fluid in horizontal Hele-Shaw cells with obstacles distributed as in the Sierpinski carpets was studied in Ref. [11] and Darcy-like flow in media formed by sequences of Menger sponges was studied in Ref. [16. In both cases, good agreement with simulation results or with scaling approaches were obtained. Hence it is expected that the scope of the work herein will benefit our understanding of transport in porous materials of current geological and technological interest.

This work is organized as follows. In Sec. III the RWI model and the basic quantities to be measured are presented, and the example of infiltration in two dimensions with a linear boundary is discussed. In Sec. III we study RWI with localized sources in two- and three-dimensional homogeneous media and in fractal lattices embedded in two and three dimensions, and establish the connection with recurrence properties of random walks. In Sec. IV] we study fluid infiltration in homogneous media and in a fractal. In Sec. V], we summarize our results and present our conclusions.

\section{RANDOM WALK INFILTRATION}

\section{A. Model definition}

RWI is defined as a process in which particles are released from a static source with constant concentration and in which those particles execute random walks in a lattice with excluded volume interaction. We denote as $a$ the edge of a lattice site, which can be occupied by at most one particle at a time. In the time unit $\tau$, each particle attempts to hop to a randomly chosen nearest neighbor $(\mathrm{NN})$ site; the hop is allowed only if that site is empty, otherwise the particle does not move. The diffusion coefficient of a free particle in a free lattice of dimension $d$ is

$$
D=\frac{a^{2}}{2 d \tau}
$$

Figs. 2(a),(b) illustrate the RWI in one dimension with a point source at $x=0$ and all sites with $x>0$ empty at $t=0$. As the particle at the source moves to $x=a$, another particle immediately refills the source [Fig. 2(a)]; in this situation, only the particle at $x=a$ can move and the only hop allowed for this particle is to the right. Fig. 2(b) shows a configuration after several particles have entered the lattice, in which only the particles at large distance from the origin can move. The particle at the source is also unable to move in this situation; it will move only when the site at $x=a$ is vacant, which requires diffusion of several intermediate particles. (a)

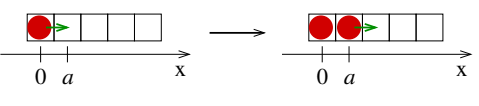

(b)

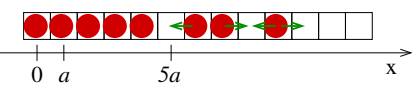

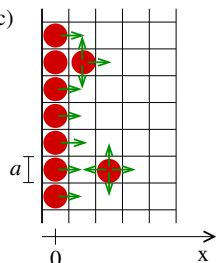

FIG. 2. (a) Initial configurations of RWI in a line with a particle at the source $x=0$. A new particle is inserted at the origin as soon as the initial particle moves to the neighboring site. (b) Configuration developed after some time, in which the particle at the source is blocked and the closest vacancy is at $x=5 a$. (c) Configuration after a few steps of RWI in a square lattice with a line source at $x=0$. The arrows indicate the possible hops of the infiltrated and of the source particles.

An equivalent model is RWI in the region $x>0$ of a square lattice with a line source at $x=0$, as shown in Fig. 2(c). Again, when one particle leaves the line $x=0$, another particle is immediately added to that point.

For the simulation of RWI, we adopt an algorithm in which the particles that attempt to hop are sequentially chosen at random from a list that includes all infiltrated particles and all particles at the source. Thus, there is no preferential choice of particles at the front or at the source for the first hop attempts. This brings to the 
model an additional randomization factor to that of the choice of the hop directions.

\section{B. Continuous limit}

Let $\rho(\vec{r}, t)$ be the particle density at position $\vec{r}$ at time $t$, given in number of particles per lattice site. Here we derive an equation for its evolution in the continuous limit.

For simplicity, consider the one-dimensional case shown in Figs. 2(a),(b). The density at position $x$ changes from time $t-\tau$ to time $t$ as the master equation

$$
\begin{aligned}
\rho(x, t)-\rho(x, t-\tau)= & \frac{1}{2} \rho(x-a, t-\tau)[1-\rho(x, t-\tau)] \\
& +\frac{1}{2} \rho(x+a, t-\tau)[1-\rho(x, t-\tau)] \\
& -\frac{1}{2} \rho(x, t-\tau)[1-\rho(x-a, t-\tau)] \\
& -\frac{1}{2} \rho(x, t-\tau)[1-\rho(x+a, t-\tau)] .
\end{aligned}
$$

In the right rand size of Eq. (3), the first and second terms account for the hops from neighboring sites to the position $x$, which are possible only if this site is not occupied; the third and fourth terms account for the hops from site $x$ to neighboring sites, which are possible only if those sites are not occupied. The joint probabilities in Eq. (3) were factorized, which considers that the densities at neighboring positions are uncorrelated; this assumption is similar to the independent cluster approximation which is also applicable for problems of diffusion, aggregation, and fragmentation, with or without particle injection [17, 18. This assumption cancels the crossed terms in the master equation. In the continuous limit, we consider $\tau \frac{\partial \rho}{\partial t} \approx \rho(x, t)-\rho(x, t-\tau)$ and $\frac{a^{2}}{2} \frac{\partial^{2} \rho}{\partial x^{2}} \approx$ $[\rho(x-a, t-\tau)-2 \rho(x, t-\tau)+\rho(x+a, t-\tau)]$, which give

$$
\frac{\partial \rho}{\partial t}=D \frac{\partial^{2} \rho}{\partial x^{2}},
$$

where $D$ is given in Eq. (2). This is the one-dimensional diffusion equation. Generalization of the above arguments to higher dimensions is straightforward, which leads to

$$
\frac{\partial \rho}{\partial t}=D \nabla^{2} \rho
$$

The boundary conditions are $\rho=1$ at the source at all times and $\rho(\vec{r}, t=0)=0$ at all points but the source.

Within the approximations in Eq. (3), the excluded volume condition of the RWI model does not affect the collective diffusion coefficient $D$ because the frustrated hop of a particle in one direction is compensated by the frustration of the hop of another particle in the opposite direction. However, if the tracer diffusion coefficient is measured (i. e. the coefficient characterizing the motion of a particular particle), it is expected to be smaller than $D$ due to the frustration of many hop attempts.

This continuous limit is expected to be valid only for $t \gg \tau$, where average particle displacements are much larger than the lattice constant $a$. In this case, the density $\rho(\vec{r}, t)$ slowly varies in time and we can compare RWI data with the solution of the diffusion equation, if available.

\section{Basic quantities}

In RWI, we denote as $I(t)$ the number of infiltrated sites of the lattice at time $t$; this quantity is hereafter termed the infiltration. In the continuous description, $I$ is obtained by integration of the density $\rho$ through the available space. In one, two, and three-dimensions, $I$ respectively represent the total length, area, and volume infiltrated by the random walkers. The infiltration is expected to scale as a power law in time with exponent $n$, in a similar fashion the fluid infiltration in Eq. 11) (a discussion on the relation between RWI and fluid infiltration is postponed to Sec. IV].

We also calculate the root mean square (rms) distance of the infiltrated particles from the source, $s_{R M S}(t)$; see illustration in Fig. 1(a). In RWI, a large fraction of the infiltrated particles move in regions with low density, so that $s_{R M S}$ is expected to follow the same scaling law of free random walks in that medium:

$$
s_{R M S} \sim t^{1 / D_{W}},
$$

where $D_{W}$ is the random walk dimension. In Euclidean lattices, we have normal diffusion with $D_{W}=2$, while in fractal lattices we expect subdiffusion with $D_{W}>2$.

If the medium has a fractal dimension $D_{F}\left(D_{F}=d\right.$ in the cases of Euclidean lattices in $d$ dimensions), then we define a characteristic infiltrated size $S$ by the equation

$$
I=S^{D_{F}} .
$$

$S$ may be interpreted as a characteristic radius of a region in which all infiltrated particles are symmetrically located around the source with no empty site between them. In fluid infiltration, the radius of the infiltrated region has the same scaling as $S$ (Sec. IV). Figs. 1(a),(b) illustrate this definition in effectively one-dimensional infiltration problems.

The quantities calculated here are presented in terms of the dimensionless time

$$
T \equiv \frac{t}{\tau}
$$

This is the average number of hop attempts of a particle that was located at the infiltration boundary at $t=0$. 


\section{Example: RWI in a square lattice with a line source}

Figs. 3(a) and 3(b) show configurations of an infiltrated square lattice at $T=1000$ and $T=4000$, respectively, considering a line source at $x=0$. Figs. 3(c) and 3(d) show the corresponding density distributions. In each time, the numerical data are averages over 20 configurations and source length $1000 a$. Figs. 3(c) and 3(d) also show the exact solution of Eq. (4), $\rho(x, t)=1-\operatorname{erf}[x /(2 \sqrt{D t})]$, where $\operatorname{erf}(u)$ denotes the error function of $u$. (a)
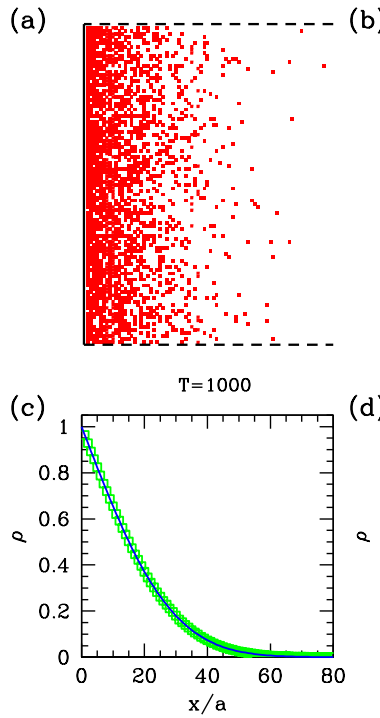

(d)

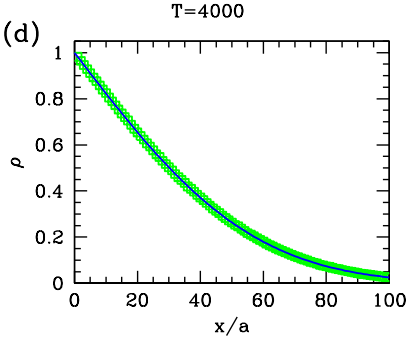

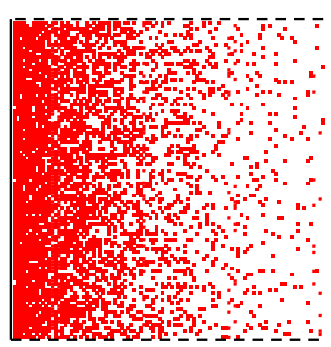

$=4000$

$\mathrm{x} / \mathrm{a}$
FIG. 3. (a), (b) Sections of size 100a of an infiltrated square lattice with a line source at the left in the two indicated times. (c), (d) The corresponding concentration distributions calculated numerically (green squares) and analytically (blue curves).

There is a very good agreement between RWI data and the solution of the diffusion equation in this case. This was formerly noted in Ref. [14. Discrepancies between the RWI and the continuous model are observed only at positions $x$ larger than $\sqrt{D t}$ by a factor of order 10 [not shown in Figs. 3(c),(d)], since the diffusion equation fails to represent the RWI in these regions (e. g. the infinitely long empty region in RWI due to the finite particle velocity).

The infiltration in this geometry scales as $I \sim T^{1 / 2}$ and Eq. (7) implies that the characteristic infiltration length also scales as $S \sim T^{1 / 2}$. This is the same scaling observed for the rms distance $s_{R M S}(t)$.

\section{E. Interpretation and relation with other models}

RWI may represent the diffusive motion of a chemical species in a fluid that fills the pores of a medium in contact with a reservoir (the source) of that species. This interpretation suggests the application of the model to infiltration of solutes in rocks or soils in cases where advective transport is negligible. A particle in this model is not necessarily equivalent to a solute molecule, but may represent a large number of molecules that entered the medium; in this case, the presence or absence of a particle represents a local solute concentration above or below a given threshold, respectively. Consider, for instance, the application of this molecular interpretation to the configuration of Fig. 2(b), in which particle hops from the source are not allowed due to excluded volume effect. It represents a case in which the region of the medium near the reservoir has a density of molecules approximately equal to that of the reservoir. In lengthscales smaller than the size of a lattice site, molecular diffusion has not ceased inside and outside the medium, but the net flux from the source to the medium is zero.

The infiltrated region has many empty sites (vacancies) in the neighborhood of the infiltrated particles, as illustrated in Figs. 3(a) and 3(b) for the case of a line source in two dimensions. The random walks of particles are equivalent to random walks of these vacancies, with excluded volume conditions and with the same diffusion coefficient $D$. When a vacancy reaches the source, it is immediately annihilated; this corresponds to the instantaneous refilling of the source in RWI. Thus, from the point of view of vacancy diffusion, we have a classical trapping problem [19, 20]. Letting $\sigma \equiv 1-\rho$ be the density of vacancies, it also obeys the diffusion equation (5); however, the boundary condition is $\sigma=0$ at the source.

The one-dimensional version of RWI [Fig. [2(a)] is equivalent to the trapping of the vacancies by a static trap at the origin. This model was originally proposed by Weiss, Kopelman, and Havlin 21. The increase of the infiltration as $t^{1 / 2}$, which is observed in RWI, is equivalent to the trapping rate decay as $t^{-1 / 2}$. Despite the apparent simplicity, that trapping model has some nontrivial features; for instance, the distance from the origin of the nearest vacancy increases anomalously as $t^{1 / 4}[21$. Similar anomalies were recently observed in other trapping models [22].

The square lattice version of RWI [Fig. 2(c)] was first proposed by Sapoval, Rosso, and Gouyet [14 for investigating the properties of the interface of a conducting cluster formed by the particles connected to the source. Subsequently, it motivated the proposal of the gradient percolation problem [23, 24]. In a recent work, RWI in deterministic fractals was studied, also with the sources at flat boundaries [15, which helped to understand scaling properties of infiltration models. 


\section{RWI WITH LOCALIZED SOURCES}

Here we consider the RWI models defined in Sec. IA with a point source localized in the neighborhood of an impenetrable boundary, as illustrated in Figs. 4(a) $(d=$ $2)$ and $4(\mathrm{~b})(d=3)$. This geometry is chosen to represent a large porous medium with a narrow hole at a surface which is in contact with a reservoir of the solute. The same geometry is also considered in simulations of RWI in determininistic fractals embedded in two- and threedimensional lattices. (a)

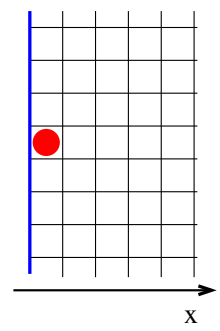

(b)

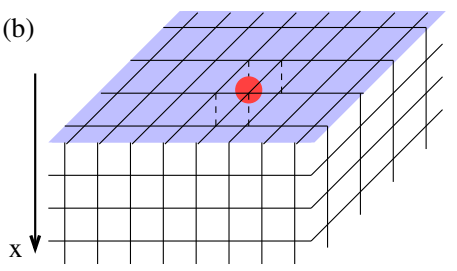

FIG. 4. Initial condition of RWI with a point source (red circle and sphere) and an impenetrable boundary at $x=0$ (blue) in (a) two and (b) three dimensions.

For simplicity, solutions of the diffusion equation (5) will be obtained with the source in media that are infinitely large in all directions (i.e., without the impenetrable boundary). This is expected to provide the same time scaling of the relevant quantities.

\section{A. Infiltration in homogeneous media}

\section{Two dimensions}

In this case, an equivalent diffusion model considers an infinite line source of radius $a$ and constant density $\rho(r \leq a, t \geq 0)=1$ placed in an infinite threedimensional domain initially with $\rho(r>a, t=0)=0$. The subsequent change in the density in a plain of fixed $z$ is governed by the diffusion equation

$$
\frac{\partial \rho}{\partial t}=\frac{D}{r} \frac{\partial}{\partial r}\left(r \frac{\partial \rho}{\partial r}\right), \quad r>a, \rho(r \rightarrow \infty, t)=0 .
$$

Ref. 25] (pp. 87-88) provides a closed solution for this problem, from which we can calculate the amount fluxed into the region $r>a$ per unit area and time, $F \equiv$ $-D\left(\frac{\partial \rho}{\partial r}\right)_{r=a}$. Assuming for convenience that $D=1 / 4$ and $a=1$ [which leads to $T=t$ in Eq.(8)], the solution for large times is

$F=\frac{1}{2}\left[\left(\frac{1}{\ln T-2 \gamma}-\frac{1}{(\ln T-2 \gamma)^{2}}\right)+\frac{1-\gamma}{(\ln T-2 \gamma)^{2}} \ldots\right]$

where $\gamma=0.57722$ is Euler's constant and $T$ is defined in Eq. 8). From this we can calculate the infiltration through a simple integration in time; this is denoted as $I_{D E}$ to stress that it results from the solution of a diffusion equation. Integrating the first bracketed term on the right hand side of Eq. (10), the infiltration in the half plane $x \geq 0$ [as in the RWI geometry of Fig. 4(a)] is, to leading order,

$$
I_{D E}(t)=\pi \int_{0}^{t} F d T=\frac{\pi}{2} \frac{T}{\log (T)-2 \gamma} .
$$

We performed simulations of RWI in square lattices of lateral size $L=2000$ with a point source in the middle of a border up to $T=2 \times 10^{4}$, which led to the injection of more than $3 \times 10^{3}$ particles. The number of configurations used to calculate $I$ and $s_{R M S}$ was $10^{3}$ (i. e. this is the number of times in which particles were injected in an empty lattice).

Fig. 5(a) shows $I$ (the number of infiltrated lattice sites) as a function of $T / \ln T$. The inset of Fig. 5(a) shows that the data obtained in two different configurations are very close to each other. This means that the accuracy of the averaged data is very high, so that the standard deviations in the main plot are much smaller than the size of the data points. The excellent linear fit in that plot confirms that $I$ has the same logarithmic corrected time scaling predicted by the continuous model [Eq. (11)]. Fig. 5(b) shows a bilogarithmic plot of $s_{R M S}$ as a function of $T$, which confirms the diffusive increase of that average displacement; the accuracy was similar to that of $I$.
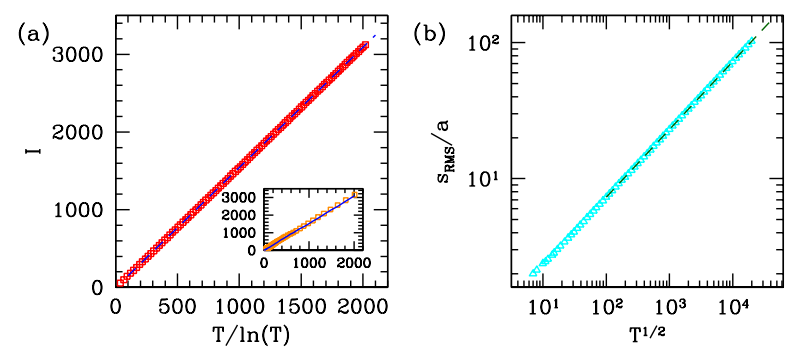

FIG. 5. RWI from a point source in two dimensions: (a) infiltration versus $T / \ln T$ (red squares) and a linear fit of the data (dashed blue line); (b) rms distance from the source versus $T^{1 / 2}$ (blue triangles) and a linear fit of the data (dashed green line) whose slope is 0.499 . The inset in (a) compares results in two configurations (blue line and orange squares).

The characteristic length of the infiltration [Eq. (7)] scales as $S \sim(T / \ln T)^{1 / 2}$. Thus, it is smaller than the rms distance $s_{R M S}$ due to the logarithmic correction.

\section{Three dimensions}

In this case, the equivalent diffusion model has a spherical point source of radius $a$ and constant density $\rho(r \leq a, t>0)=1$, placed in a infinite domain initially 
with $\rho(r>a, t=0)=0$. The subsequent change in the potential in $r>a$ is governed by the diffusion equation

$$
\frac{\partial \rho}{\partial t}=\frac{D}{r^{2}} \frac{\partial}{\partial r}\left(r^{2} \frac{\partial \rho}{\partial r}\right), \quad r>a, u(r \rightarrow \infty, t)=0
$$

The analytical solution (Ref. [25], pp. 102-103) is

$$
\rho=\frac{a}{r} \operatorname{erfc}\left(\frac{r-a}{2 \sqrt{D t}}\right)
$$

The amount fluxed into the region $r>a$ per unit area and time is given as

$$
F=-D\left(\frac{\partial u}{\partial r}\right)_{r=a}=\frac{D}{a}+\sqrt{\frac{D}{\pi t}}
$$

From this we can calculate the infiltration $I_{D E}$ through a simple integration in time. Considering $D=1 / 6$ and $a=1$ [which leads to $T=t$ in Eq.(8)] and integrating only in the region $x \geq 0$ [as in Fig. $4(\mathrm{~b})$ ], we obtain

$$
I_{D E}=2 \pi \int_{0}^{t} F d t=\frac{\pi}{3} T+\frac{4 \sqrt{\pi}}{\sqrt{T}}
$$

Our simulations of RWI were run up to $T=2 \times 10^{4}$ in a lattice with lateral size $L=700 ; I$ and $s_{R M S}$ were averaged over $10^{3}$ configurations, which also provides these quantities with high accuracy. Approximately $10^{4}$ particles were injected in each configuration.

Fig. 6(a) shows $I$ as a function of $T$; the linear fit confirms that it has the same scaling as $I_{D E}$ in Eq. (15). The characteristic length of the infiltration [Eq. (7)] consequently scales as $S \sim T^{1 / 3}$. Fig. 6(b) shows a bilogarithmic plot of $s_{R M S}$ as a function of $T$, which confirms the diffusive increase of that average distance. In this case, we also observe that the characteristic length $S$ defined from the infiltrated volume does not have the diffusive scaling of the rms distance from the source.
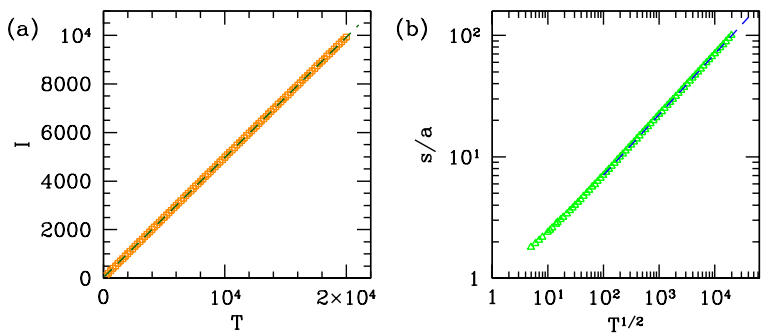

FIG. 6. Results for infiltration from a point source in three dimensions: (a) infiltration versus $T$ (orange squares) and a linear fit of the data (dashed green line); (b) rms distance from the source versus $T$ (green triangles) and a linear fit of the data (dashed blue line) whose slope is 0.500 .

\section{B. Scaling approach for the infiltration}

Here we explore the observation of Sec. IIE that RWI is equivalent to the problem of trapping of vacancies at the source. It implies that the infiltration rate $\frac{\mathrm{d} I}{\mathrm{~d} t}$ is equal to the probability $P_{\text {trapping }}(t)$ of a vacancy to be trapped at the source at time $t$. This is the probability that the first passage of the vacancy at the source occurs at time $t$. For long times, it is independent of the source position, but it is strongly dependent on the spatial dimension [20, 26].

$I$ is obtained by the integration of the first passage probability. Since this probability can be obtained as a property of a single random walk, $I$ is equal to the sum of this probability over the hops of a single walker up to time $t$. When the walker hops to a site for the first time, the number of distinct visited sites increases by one unit; thus, $I(t)$ equals the number of distinct sites visited by the walker in the medium up to time $t$, which is denoted as $N_{D}(t)$ [20, 26]. The infiltration exponent $n$ in Eq. (1) is the same exponent of the time scaling of the number of distinct sites visited by a random walker; an amplitude relating $I$ and $t^{n}$, which is omitted in Eq. (1), may depend on the boundary conditions.

In two dimensions, the dominant term in the scaling of the number of distinct sites visited by a random walker is $N_{D} \sim t / \ln t$ [26]; this agrees with the simulation result for the RWI in Sec. III A 1. In three dimensions, the dominant term is $N_{D} \sim t$ [26], which also agrees with our simulation results for RWI (Sec. III A 2).

These results establish a relation between RWI and recurrence properties of lattice random walks. Moreover, since the time evolution of the infiltration in RWI may also be predicted by the diffusion equation with constant concentration at the source, our approach also connects the solution of this equation with the recurrence properties of random walks.

\section{Infiltration in Sierpinski carpets}

Here we study RWI infiltration with localized sources in fractals embedded in two dimensions combining the previous scaling approach, numerical results of RWI, and results of numerical integration of the diffusion equation.

The iterative construction of a Sierpinski carpet is illustrated in Fig. 7(a). We denote as $b$ the scaling factor of the carpet and $m$ the number of subsquares removed in the first iteration of the construction. At each stage of construction, each subsquare is replaced by the generator (stage $n=1$ ). The removed subsquares represent the impenetrable (solid) part of the medium and the empty subsquares form the porous medium. The length of an outer border of the carpet after $n$ iterations is $L=b^{n}$. The fractal is obtained after an infinite number of iterations, with fractal dimension $D_{F}=\ln \left(b^{2}-m\right) / \ln b$. The initial condition for RWI in a carpet with a point source is shown in Fig. 7(b). 
(a)

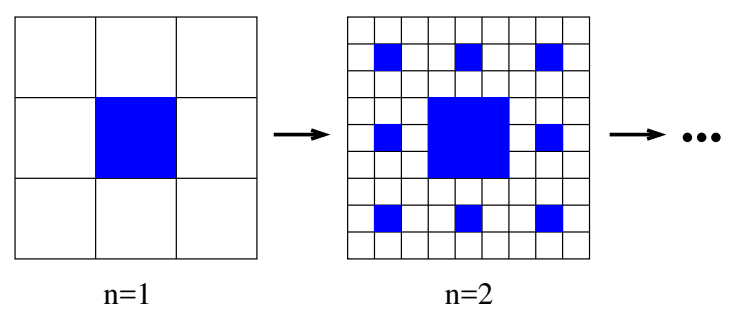

(b)

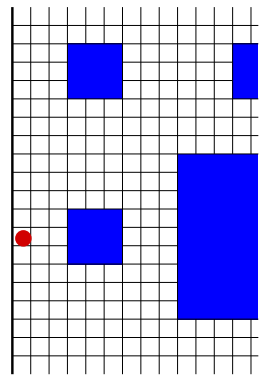

(c)

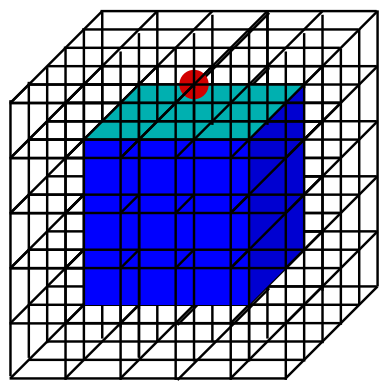

FIG. 7. (a) First two stages of construction of the carpet with $b=3$ and $m=1$, with the impenetrable solid in blue. (b) Initial condition for RWI in a carpet with a point source (red) at an outer boundary. (c) Initial condition for RWI in a sponge with a point source (red) at an outer boundary.

The number of distinct sites visited by a random walker in this fractal is expected to scale as

$$
N_{D} \sim t^{D_{S} / 2} \quad, \quad D_{S}=2 \frac{D_{F}}{D_{W}},
$$

where $D_{S}$ is the spectral (or fracton) dimension. Eq. (16) is known as Alexander-Orbach (AO) relation [19, 27]; it is based on the assumption of homogeneous distribution of the random walker position within the accessible fractal volume at time $t$. However, the $\mathrm{AO}$ relation has been criticized in the past due to deviations observed in some numerical results; for a discussion, see Ref. [19, Ch. 7, and Ref. [28].

For RWI, Eqs. (1) and (16) give the infiltration exponent $n=D_{F} / D_{W}$. In the carpet of Fig. 77(a), $D_{F}=\ln 8 / \ln 3=1.8928 \ldots$ Ref. [29] conjectured that the exact value of the random walk dimension in this carpet is $D_{W}=2+\ln (10 / 9) / \ln 3=2.0959 \ldots$; this value agrees with the numerical estimates 2.101(22) [30 and 2.106 (16) [31. Using the exact $D_{F}$ and the conjectured $D_{W}$, we obtain $n=0.903 \ldots$.

We performed simulations of RWI in the $7^{\text {th }}$ stage of construction of this carpet up to $T=10^{5}$. Averages were also taken over $10^{3}$ configurations, with more than $10^{4}$ injected particles in each one; thus, the accuracy of the data is similar to that in Fig. 5.

In Fig. 8(a), we show a bilogarithmic plot of $I$ versus $T$ in this fractal. The slope at long times is still slightly smaller than the proposed value of $n$. This is consistent with previous calculations of $N_{D}$ in the carpets, which suggested corrections to the AO relation [32, 33. In Fig. 8 (b), we show the ratio $I / T^{0.903}$ as a function of $1 / T^{0.27}$;

the variable in the abscissa is the one that provides the best linear fit of the data in the range $5 \times 10^{2} \leq T \leq 10^{5}$ (among other variables in the form $T^{-\lambda}$ with positive $\lambda$ ). The convergence to a finite value at long times confirms that the infiltration scales asymptotically with the proposed value of $n$, and the small exponent in the abscissa of Fig. 8(b) indicates the presence of large subdominant corrections to the $\mathrm{AO}$ relation.
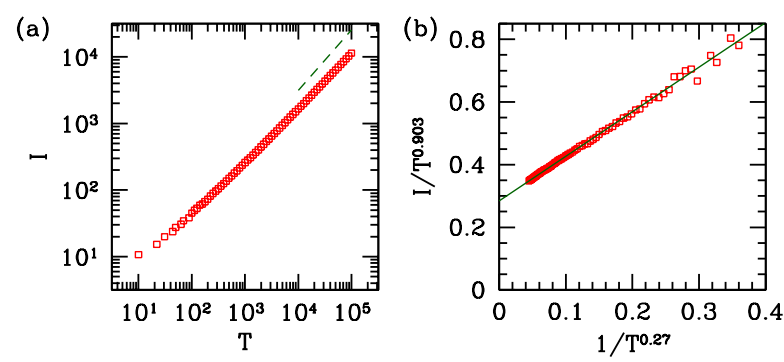

FIG. 8. (a) Time evolution of the infiltration from a point source in the SC with $b=3$ and $m=1$. The dashed line has the theoretically predicted slope 0.903 . (b) Scaled infiltration as a function of $1 / t^{0.27}$ for RWI in the Sierpinski carpet. The solid line is a linear fit of the data for $5 \times 10^{2} \leq T \leq 10^{5}$.

For comparing different methods of solution for the RWI problem, we also solved Eq. (5) numerically. We use a time explicit finite-difference control volume solution for the solute infiltration from a point source $(\rho=1)$ into the stage $n=3$ of the same Sierpinski carpet [Fig. 7(a)], with diffsuivity $D=1$ at the empty subsquares and with the solid squares representing areas of very low diffusivity $D<<1$. The numerical solutions, similar to that outlined in [34, involves a $3^{6} \times 3^{6}$ mesh of square node-centered control volumes of side length $\Delta=1 / 3^{6}$ and, to retain stability of the explicit scheme, a time step $\Delta t=0.125 \times \Delta^{2}$.

Fig. 9(a) shows some concentration contours after 120,000 time steps. The measure over time of this simulation is the solute content $I_{D E}$, see Eq. (11), from which we obtain the local time exponent $n=d \ln I_{D E} / d \ln t$. The key observation in Fig. 9(b) is that this exponent approaches, in long times, a consatnt value that is within fractions of a percent of the expected value of $\sim 0.903$. Consequently, this solution significantly improves that of direct simulation of RWI (Fig. 8). More importantly, it confirms with very good accuracy that the AO relation is valid in a Sierpinski carpet, advancing over the current and previous random walk based methods.

The oscillations observed in Fig. 9(b) at long times resemble the log-periodic oscillations shown in Refs. [35, 36 in models of random walks in regular fractals. Some methods were developed to treat data with this type of oscillation 35, 37, which may be helpful whenever accurate exponent estimates are not available.

In Ref. [15], a scaling approach was used to relate the infiltration exponent $n$ [Eq. (1)] and the random walk dimension $D_{W}$ in cases where the source was one 

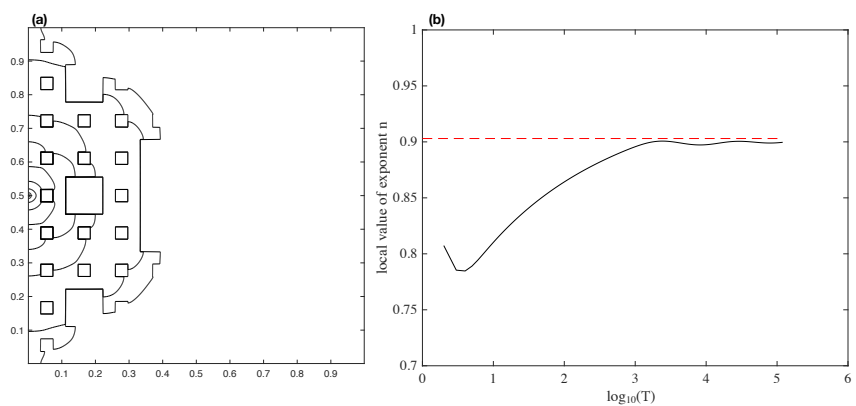

FIG. 9. Solute diffusion into a $3^{\text {rd }}$ stage Sierpinski carpet with $b=3, m=1$. (a) The concentration contours after 120,000 time steps: from right to left, $\rho=0.0 .005,0.01,0.05$, and from 0.1 to 0.9 in intervals of 0.1 . Solid squares and pores are not distinguished by different colors here. (b) The local time exponent $n=d \ln I_{D E} / d \mathrm{l} n t$; the dashed line has the theoretically predicted value 0.903 .

of the outer boundaries of the fractal. For a border with dimension $D_{B}$, it was shown that

$$
n=\frac{D_{F}-D_{B}}{D_{W}} \text {. }
$$

This result may be extended to infiltration from a point source in a carpet because, in this case, $D_{B}=0$, which gives $n=D_{F} / D_{W}$.

\section{RWI in Menger sponges}

A Menger sponge is iteratively constructed here by extending to three dimensions the procedure used to construct the SCs: first, a cube is divided in $b^{3}$ subcubes and $m$ symmetrically distributed subcubes are removed, which forms the generator; each one of the subcubes is then divided following the same rule, and this process is repeated indefinitely. The fractal dimension of the sponge is $D_{F}=\ln \left(b^{3}-m\right) / \ln b$.

Here we study RWI in the sponge with $b=5$ and $m=27$. The initial condition for RWI with a point source in this sponge is illustrated in Fig. 77(c). The fractal dimension is $D_{F}=\ln 98 / \ln 5 \approx 2.84880$ and the estimate of the random walk dimension is $D_{W}=2.09(6)$ [15.

These values imply $D_{F}>D_{W}$. The AO relation [Eq. [16] ] is expected to be valid only when random walks are recurrent in a given fractal, but this is not the case here. Instead, here the accessible region for the infiltrated particles has a radius $\sim s_{R M S}$ and a volume of order $s_{R M S}^{D_{F}} \sim t^{D_{F} / D_{W}} \gg t$, i.e. this region is much larger than the number of sites that the walker can visit within a time $t$. This means that only a small fraction of the accessible sites is visited, and this fraction tends to zero asymptotically. In such cases, we expect that $N_{D}$ scales linearly in time, and so $I$ is also linear in time.

We simulated RWI in the $4^{\text {th }}$ stage of construction of the sponge up to $T=2 \times 10^{4}$. Averages were taken over $10^{3}$ configurations and more than $1.5 \times 10^{4}$ particles were injected in each configuration. Fig. 10 shows that the infiltrated volume $I$ increases linearly in time in all the simulated time interval, which confirms the above prediction. Note that the same scaling was obtained in a homogeneous three-dimensional medium with a point source, as shown in Sec. III A 2.

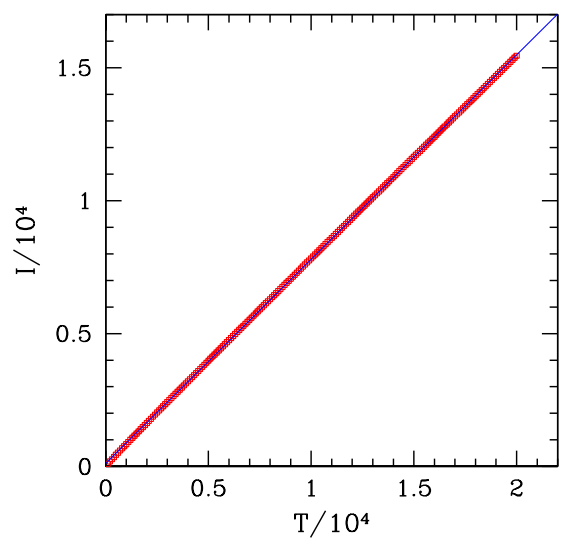

FIG. 10. Infiltrated volume as a function of time for RWI in the Menger sponge. The solid line is a linear fit of the data.

It is interesting to observe that the infiltration in this fractal is normal, although the diffusion of a single particle is clearly anomalous $\left(D_{W}>2\right)$. The same observation is applicable to other fractals where random walks are not recurrent, i.e. $D_{W}<D_{F}$. Consequently, in an infiltration experiment on such a fractal with a localized source, the measurements of infiltrated mass or volume will not show any anomaly, but the same linear increase observed in a homogeneous medium. In order to observe the anomaly, it is necessary to measure the diffusion lengths of the infiltrated molecules (or of other tracer particles).

RWI in the sponge also shows a failure of the scaling relation (17), since that relation implies the $\mathrm{AO}$ relation for $D_{B}=0$. Indeed, Eq. (17) was obtained in Ref. [15] under the assumption that a finite fraction of a region of lateral size $s_{R M S}$ was filled with infiltrated particles. This is actually the case in two-dimensional systems with linear sources or three-dimensional systems with planar sources. However, in a fractal with large $D_{F}$, a point source is too small to fill a finite fraction of a region with that size.

\section{RELATION WITH FLUID INFILTRATION}

Here we consider infiltration of a fluid with a constant pressure head $h=1$ at a fixed boundary, which is the source of the fluid molecules, and assume that the head vanishes at the infiltration front. In the region limited 
by these boundaries, the slowly varying pressure head $h$ obeys the Laplace equation

$$
\nabla^{2} h=0,
$$

and the velocity of the infiltration front obeys the Darcy law

$$
\vec{v}=-K \vec{\nabla} h,
$$

where $K$ is the hydraulic conductivity. For simplicity, we consider $K=1$ in a homogeneous medium.

This model was numerically studied in several carpets with different boundaries in Refs. 34] and [38. The estimates of the infiltration exponent $n$ were in excellent agreement with estimates from the RWI model and with the scaling relation (17) 15]. Experiments with infiltration in finite stages of construction of some carpets also showed good agreement with those estimates [11.

Here we extend this comparison to infiltration with localized sources.

\section{A. Two dimensions and localized source}

We consider the problem in a two-dimensional cylindrical geometry, where a small source is located at $r=a$. Eq. 18 becomes

$$
\frac{\partial}{\partial r}\left(r \frac{\partial h}{\partial r}\right)=0, \quad h(a)=1, h(R)=0,
$$

which gives the head profile

$$
h=\frac{\ln r-\ln R}{\ln a-\ln R}, \quad a \leq r \leq R .
$$

Eq. 19 for the moving front becomes

$$
-\left(\frac{\partial h}{\partial r}\right)_{r=R}=\frac{d R}{d t}
$$

and substitution of Eq. 21) gives

$$
\frac{d R}{d t}=-\frac{1}{R(\ln a-\ln R)}, \quad R(0)=a
$$

The solution for $R(t)$ is

$$
\frac{R^{2}}{2}(\ln R-\log a)+\frac{a^{2}-R^{2}}{4}=t
$$

In limit of $R>>$ a, i.e. when the front displacement is much larger than the radius of the source, we have

$$
R \approx 2\left(\frac{t}{\ln t}\right)^{\frac{1}{2}}
$$

The infiltrated area in this limit is

$$
A \approx 4 \pi\left(\frac{t}{\ln t}\right) .
$$

This is the same linear increase depleted by a logarithmic correction that we observed in RWI (Sec. III A 1). The front radius $R$ is equivalent to the length $S$ defined for RWI [Eq. (7)], and $R$ may be directly measured in an experiment.

\section{B. Three dimensions and localized source}

The model can be extended to three dimensions, where Eq. 18 for the head leads to

$$
\frac{\partial}{\partial r}\left(r^{2} \frac{\partial h}{\partial r}\right)=0, \quad h(a)=1, h(R)=0
$$

with the same moving front condition as in the cylindrical case

$$
-\left(\frac{\partial h}{\partial r}\right)_{r=R}=\frac{d R}{d t} .
$$

The solution of Eq. 27) in $a \leq r \leq R$ is

$$
h=\frac{1}{r} \frac{a R}{R-a}-\frac{a}{R-a} .
$$

Substituting Eq. 29 in Eq. 28), we obtain the equation for the movement of the front as

$$
\frac{d R}{d t}=\frac{a}{R^{2}-a R}, \quad R(0)=a .
$$

The solution for $R(t)$ is

$$
\frac{R^{3}}{3 a}-\frac{R^{2}}{2}+\frac{a^{2}}{6}=t
$$

In limit where the front displacement is much larger than the source radius, $R>>a$, we have

$$
R \approx(3 a t)^{1 / 3}
$$

and the infiltrated volume is

$$
V \approx 4 \pi a t
$$

Here, the fluid infiltration shows the same linear time increase of the RWI in three dimensional homogeneous media. Note that the radius $R$, which may be experimentally measured, has a subdiffusive scaling, which contrasts the rms displacement of RWI; again, $R$ is analogous to the length $S$ defined for RWI.

\section{Extension to fractal media}

We simulated the fluid infiltration model in the $3^{\text {rd }}$ stage of construction of the carpet with $b=3$ and $m=1$ and with a point source in an external boundary using the same control volume finite difference methods of Ref. 34. Essentially, at each time step, this involves the numerical solution of $\nabla(K \nabla h)=0$, with $K=1$ in the spaces and $K<<1$ in the obstacles. In the current simulations, we use a $3^{5} \times 3^{5}$ mesh of square node centered control volumes of size $\Delta=1 / 3^{5}$ and a time step of $\Delta t=0.005$; as fully explained in Ref. [4, the accuracy of the calculation is independent of the time step size. 
In Fig. 11, we show the infiltration front at the time in which the fluid reaches the horizontal domain boundaries. In Fig. 11p, we show the time evolution of the infiltrated area. This area scales with an exponent close to the theoretical value 0.903 , which is also in agreement with that of RWI in the same carpet (Sec. III C).

In this simulation, the dimension of the gate $a$ for the infiltration is $1 / 9$ the size of the smallest obstacle dimension in the carpet; we would expect values closer to the theory as the relative size of the gate is reduced. In fact, simulations on the geometry in Fig. 11] with coarse grids and thus wider gates show a quadratic dependence between the exponent $n$ and gate size; in the continuous limit $(a \rightarrow 0)$, this quadratic fit suggests an exponent $n=0.8994$, which differs less than $0.5 \%$ from the theoretical value.
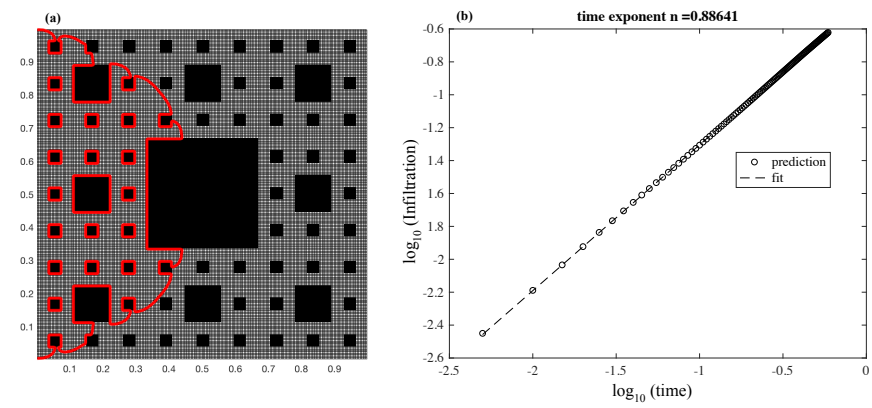

FIG. 11. Area filled with fluid as a function of time in the carpet with $b=3$ and $m=1$. (a) The infiltration front at the point the fluid reaches the horizontal boundary. (b) The log-log plot of the infiltration versus time.

\section{CONCLUSION}

We studied a random walk infiltration model with small sources at the boundaries of homogeneous and fractal media. The model represents the penetration of a solute from a reservoir in contact with a porous medium by a narrow hole, viz. the source. A scaling approach proposes that the number of infiltrated particles increases in time as the number of distinct sites visited by a single random walker in the same medium, $N_{D}(t)$. This is confirmed by numerical results obtained by direct simulations of the lattice model and by integration of the diffusion equation that represents the model in the continuous limit. In a Sierpinski carpet, the numerical integration of the diffusion equation provides an exponent for the time evolution of the infiltration which is very close to the value of the Alexander-Orbach conjecture for $N_{D}$, improving previous results obtained from random walk models. In a Menger sponge where the fractal dimension is larger than the random walk dimension, the infiltrated volume increases linearly in time, similarly to a homogeneous medium.

We also studied a fluid infiltration model in the same geometry, with a constant pressure head at the source and with the front displacement driven by the local gradient of that head. Exact and numerical solutions in two and three dimensions and in Sierpinski carpets show that the fluid infiltration model is in the same universality class of the random walk infiltration. The main difference between these models is the sharp front of fluid infiltration, while in RWI the front is diffuse; in Fig. 12, these differences are highlighted, as well as the differences between the random walk infiltration in a lattice and the solution of the corresponding diffusion equation. However, in the two models, the evolution of the fronts are driven by the gradient of two analogous fields, which are the fluid head and the particle concentration. Note that the equivalence between the two problems is observed in cases where random walks are recurrent (carpets or twodimensional media) and not recurrent (three-dimensional homogeneous media). The scaling approach then sets a relation between the different infiltration models from localized sources and the recurrence properties of random walks.

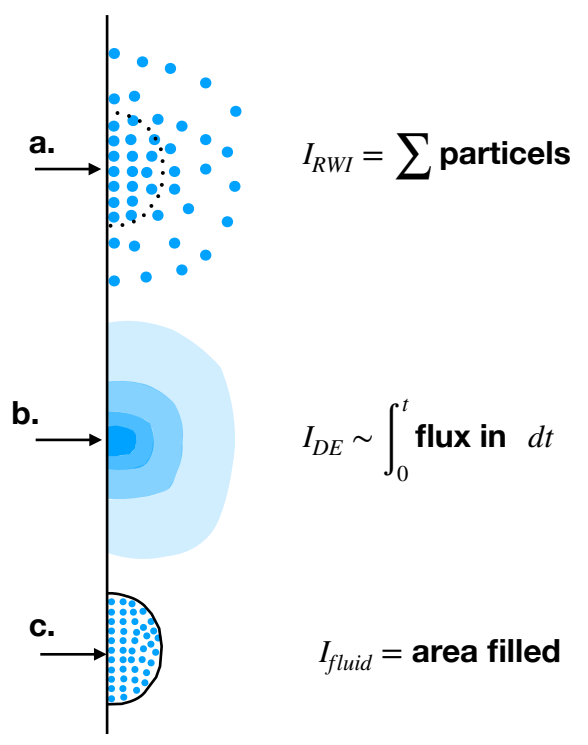

FIG. 12. Despite the different natures of the conservation of the RWI, diffusion equation, and fluid infiltration processes, they all have identical scaling, $I_{R W I} \sim I_{D E} \sim I_{\text {fluid }} \sim t^{n}$.

In fractal media where random walks are not recurrent, we show the failure of a previous exponent relation for infiltration from flat boundaries [15]. This is the case of the Menger sponge studied here and of other fractals embedded in three dimensions with the fractal dimension exceeding the random walk dimension (typically fractals with large dimensions and weak subdiffusion). In these cases, the normal infiltration scaling of three dimensional systems, $I \sim t$, is obtained, which hides the fractality of the infiltrated medium.

As a final note, we recall that normal diffusion was already observed in several fractals, i.e. $D_{W}=2$ 39 41. However, infiltration may be anomalous in those systems 
because the exponent $n$ also depends on the dimension of the medium and on the dimension of the source, as shown here for the case of small localized sources and in previous works for extended sources.

\section{ACKNOWLEDGMENTS}

FDAAR acknowledges support by the Brazilian agencies CNPq (304766/2014-3) and FAPERJ (E$26 / 202941 / 2015$ ) and thanks the hospitality of the Department of Civil, Environmental, and Geo-Engineering of University of Minnesota, where part of this work was done. VRV acknowledges support from the James L. Record Professorship.
[1] W. H. Green and G. Ampt, J. Ag. Sci, 4, 1 (1911).

[2] G. S. Campbell and J. M. Norman, An Introduction to Environmental Biophysics, 2nd ed. (Springer, 1998).

[3] V. R. Voller and S. Peng, Polym. Eng. Sci. 35, 1758 (1995).

[4] V. R. Voller and Y. F. Chen, Int. J. Num. Meth. Fluids 23, 661 (1996).

[5] S. D. Logsdon, Soil Sci. 162, 233 (1999).

[6] D. A. Lockington and J.-Y. Parlange, J. Phys. D: Appl. Phys. 36, 760 (2003).

[7] M. Küntz and P. Lavallée, J. Phys. D 34, 2547 (2001).

[8] M. A. Wilson, W. D. Hoff, C. Hall, B. McKay, and A. Hiley, Phys. Rev. Lett. 90, 125503 (2003).

[9] A. El Abd, J. Phys. D: Appl. Phys. 37, 2305 (2004).

[10] D. N. Gerasimov, V. A. Kondratieva, and O. A. Sinkevich, Physica D 239, 1593 (2010).

[11] N. Filipovitch, K. M. Hill, A. Longjas, and V. R. Voller, Water Resources Research 52, 5167 (2016)

[12] A. El Abd, Appl. Radiat. Isot. 105, 150 (2015).

[13] C. L. A. Berli, M. Mercuri, and M. G. Bellino, Phys. Chem. Chem. Phys. 19, 1731 (2017).

[14] B. Sapoval, M. Rosso, and J. F. Gouyet, J. Phys. (Paris), Lett. 46, L149 (1985).

[15] F. D. A. Aarão Reis, Phys. Rev. E 94, 052124 (2016)

[16] A. S. Balankin, J.-C. Valdivia, J. Marquez, and O. S. M. A. Solorio-Avila, Phys. Lett. A 380, 2767 (2016).

[17] S. N. Majumdar, S. Krishnamurthy, and M. Barma, Phys. Rev. Lett. 81, 3691 (1998).

[18] F. D. A. Aarão Reis and R. B. Stinchcombe, Phys. Rev. E 71, 026110 (2005).

[19] H. Havlin and D. Ben-Avraham, Advances in Physics 51, 187 (2002).

[20] S. Redner, A Guide to First-Passage Processes (Cambridge University Press 2, Cambridge, UK, 2001).

[21] G. H. Weiss, R. Kopelman, and S. Havlin, Phys. Rev. A
39, 466 (1989).

[22] E. G. Flekkoy, Phys. Rev. E 95, 012139 (2017).

[23] A. Bunde and J. F. Gouyet, J. Phys. A: Math. Gen. 18, L285 (1985).

[24] M. Rosso, J. F. Gouyet, and B. Sapoval, Phys. Rev. Lett. 57, 3195 (1986).

[25] J. Crank, Mathematics of Diffusion, 2nd ed. (Clarendon Press, Oxford, UK, 1975).

[26] E. W. Montroll and B. J. West, in Fluctuation Phenomena, edited by E. W. Montroll and J. L. Lebowitz (North-Holland, Amsterdam, Netherlands, 1979) pp. 62-205.

[27] S. Alexander and R. Orbach, J. Phys. Lett. (Paris) 43, L625 (1982).

[28] I. M. Sokolov, J. Phys. A: Math. Theor. 49, 095003 (2016)

[29] A. S. Balankin, Phys. Lett. A 381, 2801 (2017).

[30] F. D. A. A. Reis, Journal of Physics A: Mathematical and General 28, 6277 (1995).

[31] M. H. Kim, D. H. Yoon, and I.-m. Kim, Journal of Physics A: Mathematical and General 26, 5655 (1993).

[32] F. D. A. A. Reis, Phys. Lett. A 214, 239 (1996).

[33] R. Dasgupta, T. K. Ballabh, and S. Tarafdar, J. Phys. A: Math. Gen. 32, 6503 (1999).

[34] V. R. Voller, Water Resources Research 51, 2119 (2015)

[35] M. A. Bab, G. Fabricius, and E. V. Albano, J. Chem. Phys. 128, 044911 (2008).

[36] E. Akkermans, O. Benichou, G. V. Dunne, A. Teplyaev, and R. Voituriez, Phys. Rev. E 86, 061125 (2012).

[37] R. Haber, J. Prehl, K. H. Hoffmann, and H. Herrmann, J. Phys. A: Math. Theor. 47, 155001 (2014).

[38] F. D. A. A. Reis, D. Bolster, and V. R. Voller, Adv. Water Res. 113, 180 (2018)

[39] R. Burioni and D. Cassi, Phys. Rev. E 49, R1785 (1994).

[40] G. Forte, F. Cecconi, and A. Vulpiani, Eur. Phys. J. B 87, 102 (2014).

[41] A. S. Balankin, Phys. Lett. A 382, 1534 (2018). 